BJMG 10/2 (2007) 3-14

$10.2478 / \mathrm{v} 10034-008-0001-5$

REVIEW ARTICLE

\title{
TAUOPATHIES: A DISTINCT CLASS OF NEURODEGENERATIVE DISEASES
}

\author{
Ozansoy M, Başak AN
}

\begin{abstract}
*Corresponding Author: A. Nazlı Başak, Boğaziçi University, Department of Molecular Biology and Genetics, 34342, Bebek, Istanbul; Tel.: +212-359-66-79; Fax: +212-359-72-98; E-mail: basak@boun.edu.tr
\end{abstract}

\begin{abstract}
Neurodegenerative diseases are characterized by neuronal loss and intraneuronal accumulation of fibrillary materials, of which, neurofibrillary tangles (NFT) are the most common. Neurofibrillary tangles also occur in normal aging and contain the hyperphosphorylated microtubule-associated protein tau. A detailed presentation is made of the molecular bases of Alzheimer's disease (AD), postencephalitic parkinsonism, amyotrophic lateral sclerosis/parkinsonism-dementia complex (ALS/PDC) of Guam, progressive supranuclear palsy (PSP), corticobasal degeneration (CBD), Pick's disease, frontotemporal dementia (FTD), Down's syndrome, myotonic dystrophy (DM) and Niemann-Pick Type C (NPC) disease, which are considered to be common tauopathies. The unique human tau gene extends over 100 $\mathrm{kb}$ of the long arm of chromosome 17 and contains 16 exons. The human brain contains six tau isoforms that contain from 352 to 441 amino acids. To date, 34 pathogenic tau mutations have been described among 101 families affected by FTD with parkinsonism linked to chromosome 17 (FTDP-17). These mutations may involve alternative splicing of exon 10 that lead to changes in the proportion of 4-repeat- and 3-repeat-tau isoforms, or may modify tau interactions with microtubules. Tau aggregates dif-
\end{abstract}

\footnotetext{
* Department of Molecular Biology and Genetics, Neurodegenration Research Laboratory, Boğaziçi University, Bebek, Istanbul, Turkey
}

fer in degree of phosphorylation and in content of tau isoforms. Five classes of tauopathies have been defined depending on the type of tau aggregates. The key event in tauopathies is the disorganization of the cytoskeleton, which is based on mutations/polymorphisms in the tau gene and lead to nerve cell degeneration. In this review, tauopathies as a distinct class of neurodegenerative diseases are discussed with emphasis on their molecular pathology and genetics.

Key words: Tauopathies; Neurodegeneration; Tau gene, Tau protein, Tau mutations

\section{TAUOPATHIES: AN OVERVIEW}

Neurodegenerative diseases are characterized by neuronal loss and intraneuronal accumulations of fibrillary materials. Among several intracellular inclusions such as Hirano bodies, Lewy bodies, Pick bodies and neurofibrillary tangles (NFT), the latter are the most common [1]. They are consistently found in Alzheimer's disease (AD), postencephalitic parkinsonism, amyotrophic lateral sclerosis/parkinsonism-dementia complex (ALS/PDC) of Guam, progressive supranuclear palsy (PSP), corticobasal degeneration (CBD), Pick's disease, frontotemporal dementia (FTD), dementia pugilistica and head trauma, and Down's syndrome. They have also been described in Gerstmann-Straussler-Scheinker syndrome (GSS), Hal-lervordern-Spatz disease, myotonic dystrophy (DM), Niemann-Pick type C (NPC) disease, subacute sclerosing panencephalitis among other rare conditions, but also occur in normal aging 
and contain the hyperphosphorylated microtubuleassociated protein tau [1]. In this review, the molecular bases of AD, postencephalitic parkinsonism, ALS/PDC, PSP, CBD, Pick's disease, FTD, Down's syndrome, DM and NPC disease, all considered to be common tauopathies, will be discussed [1].

Alzheimer's Disease (AD). Alzheimer's disease is a progressive neurodegenerative disorder that leads to dementia and affects approximately $10 \%$ of the world population older than 65 years of age. Memory loss is followed by aphasia, agnosia, apraxia and behavioral disturbances [1]. Senile plaques and NFT are the main brain lesions observed in AD. The former result from extracellular accumulation of the peptide $A \beta$, derived from the $\beta$-amyloid precursor protein (APP), into amyloid deposits, which are diffusely and variably distributed throughout the cerebral cortex and in subcortical structures [2,3]. In familial $\mathrm{AD}$, mutations have been found on the APP gene [2]. The NFT consist of abnormal fibrils aggregated into paired helical filaments (PHF) and contain the hyperphosphorylated form of tau protein. They are usually observed in the large pyramidal cells of the hippocampus and entorhinal cortex, the supragranular and infragranular layers of the association cortical areas, nucleus basalis of Meynert, amygdala, locus coeruleus and dorsal raphe, the primary motor and sensory cortices being relatively spared. The demonstration of both senile plaques and NFT within these regions of the cerebral cortex is essential for a definite diagnosis of AD. However, NFT at a lower density are also present in the entorhinal cortex and hippocampus of normal elderly brains, whereas the neocortex exhibits only isolated NFT [1,3].

Postencephalitic Parkinsonism. Many patients who survived the influenza pandemic of 1916-1926 later developed postencephalitic parkinsonism with extrapyramidal symptoms as the major clinical features. Affected patients did not show any cognitive changes and were usually neither aphasic nor apraxic [1]. Immunohistochemical analysis demonstrated that NFT were present in variable density in the hippocampus and entorhinal cortex, in neocortical areas and in subcortical regions. Higher NFT densities were observed in the hippocampus (CA1 and subiculum), area 20 of the neocortex and putamen, indicating that some regions were preferentially affected by the degenerative process $[1,4]$.
Amyotrophic Lateral Sclerosis/Parkinsonism Dementia Complex (ALS/PDC) of Guam. This chronic neurodegenerative disorder is highly prevalent in the native Chamorro population of Guam in the Western Pacific [2]. Clinically, it is indistinguishable from sporadic ALS and presents with fasciculations and signs of involvement of lower and upper motor neurons. Parkinsonism-dementia is characterized by an insidious progressive mental decline and extrapyramidal signs including bradykinesia, rigidity and, less often, tremors. Both aspects of the disease are frequently associated but may occur separately. The brain shows severe cortical atrophy and neuronal loss. There is widespread NFT formation, especially in the temporal and frontal isocortex, hippocampal formation and several subcortical structures [2,5]. Immunohistochemical studies have revealed that pathological tau proteins are present in the NFT [1].

Progressive Supranuclear Palsy (PSP). This late-onset atypical parkinsonian disorder is characterized by supranuclear vertical gaze paralysis, moderate or severe postural instability with episodes of falling during the first year of onset of symptoms, and facial, nuchal and troncular dystonia; dementia is common in the later stages $[1,5,6]$. Progressive supranuclear palsy is characterized by neuronal loss, gliosis and NFT formation. Subcortical localization of NFT in basal ganglia, brainstem and cerebellum, led to PSP being considered as a model of "subcortical dementias." However, degenerative lesions have been described in the perirhinal, inferior temporal and prefrontal cortex, the density of NFT varying among cases $[6,7]$. Further studies have demonstrated that the primary motor cortex is more severely affected than the neocortical association areas when compared to $\mathrm{AD}[1,5,8]$. Glial fibrillary tangles have been detected in PSP brains [5,8-11].

Corticobasal Degeneration (CBD). This rare, sporadic and slowly progressive neurodegenerative disorder is clinically characterized by cognitive disturbances, e.g., aphasia and apraxia, and extrapyramidal motor dysfunction, e.g., rigidity, limb dystonia, akinesia and action tremor [1,5]. Sometimes moderate dementia emerges late in the course of the disease. There is clinical and pathological overlap between PSP and CBD, so that distinction of these conditions on a neuropathological basis becomes very important. Neuropathological examination shows frontoparietal atrophy of the brain and glial 
and neuronal abnormalities [5,11]. The glial pathology is dominated by astrocytic plaques, and numerous tau-immunoreactive inclusions in the white matter. The presence of achromatic ballooned neurons has been shown in cortex, brainstem and subcortical structures, as well as neuritic changes and NFT. Immunochemical studies have been given great importance in distinction of PSP and CBD [1,5,11].

Pick's Disease. This rare disorder is characterized by a distinctly progressive dementia. Early in the clinical course, there are signs of frontal disinhibition including mood disturbances and progressive language impoverishment leading to mutism [1]. Prominent frontotemporal lobar atrophy, gliosis, severe neuronal loss, ballooned neurons and the presence of neuronal inclusions, called Pick bodies, in both cortical and subcortical structures are characteristic of the disease [1,2]. Pick bodies contain tau protein and occur at higher density in the hippocampus than in the neocortex. In the former, Pick bodies are numerous in the granule cells of the dentate gyrus, in the CA1 field, the subiculum and the entorhinal cortex. In the latter, they are mainly found in layers II and VI of the anterior segment of temporal and frontal lobes $[1,2,5]$.

Frontotemporal Dementia (FTD). Historically, this has often been classified as a form of Pick's disease, even when Pick bodies were not present. However, FTD may be categorized with different subgroups. A consensus on clinical and neuropathological criteria for FTD has been published which clarified the distinction between Pick's disease and FTD [12]. An autosomal dominant disease related to FTD, characterized by adult-onset behavioral disturbances, frontal lobe dementia, parkinsonism and amyotrophy and linked to chromosome 17q21 has been described and is called FTD with parkinsonism linked to chromosome 17 (FTDP-17) [12]. Although clinical heterogeneity has been described between and within families with FTDP-17, the usual symptoms include behavioral changes, loss of frontal executive functions, language deficit and hyperorality $[12,13]$. Parkinsonism and amyotrophy have been found in a few families, but are not consistent features [14]. Brains of FTD patients show atrophy of frontal and temporal lobes, severe neuronal cell loss, grey and white matter gliosis, and superficial laminar spongiosis. An important characteristic is the filamentous pathology that affects neuronal cells and/or glial cells in some cases. Tau mutations segregate with the pathology of FTDP-17 indicating their pathogenic role [13-15].

Myotonic Dystrophy [DM]. This autosomal dominant and slowly progressive multisystemic disorder is characterized by myotonia, muscular atrophy, cataract and endocrine dysfunction. Affected individuals present with a highly variable phenotype, ranging from an asymptomatic condition to a severe congenital form. Impairment of intellectual and cognitive function in DM has been reported [16]. The molecular basis is an unstable CTG trinucleotide repeat in the 3' untranslated region (3'UTR) of a gene that encodes a putative Ser/Thr protein kinase (DM protein kinase), located on chromosome 19 [16]. There is reduced brain weight and minor abnormalities in gyral architecture, and a disordered cellular arrangement with neurons being present in subcortical white matter, and intracytoplasmic inclusion bodies in cortical and subcortical structures. The presence of abnormally frequent NFT has been reported in the temporal lobe, especially in the parahippocampal gyrus [16]. Immunoblotting has revealed taupositive inclusion bodies in the hippocampus, the entorhinal cortex and in most of the temporal areas. The amounts of tau proteins are higher in the most severely affected brains, but lower than in AD [1].

Down's Syndrome. Down's syndrome patients have numerous somatic dysfunctions that occur during development which are due to trisomy of chromosome 21. In particular, a deficit of growth and maturation of the brain is consistently described, and patients develop a variable degree of cognitive impairment, usually leading to dementia after 50 years of age [17]. Neuropathologically, severe neuronal loss has been described in the hippocampal formation, neocortex and in subcortical areas [17]. The formation of NFT and amyloid deposits occurs prior to neuronal loss. Neurofibrillary degeneration with tau accumulation appears later in life. The hippocampal formation, including the entorhinal cortex, contains the highest number of NFT. Down's syndrome brain extracts contain significant amounts of insoluble tau $[1,17]$.

Niemann-Pick Type C (NPC) Disease. This is a cholesterol storage disease with defects of intracellular trafficking of exogenous cholesterol derived from low density lipoproteins. Niemann-Pick type C includes juvenile dystonic lipidosis, ophthalmoplegic lipidosis, neurovisceral storage disease 


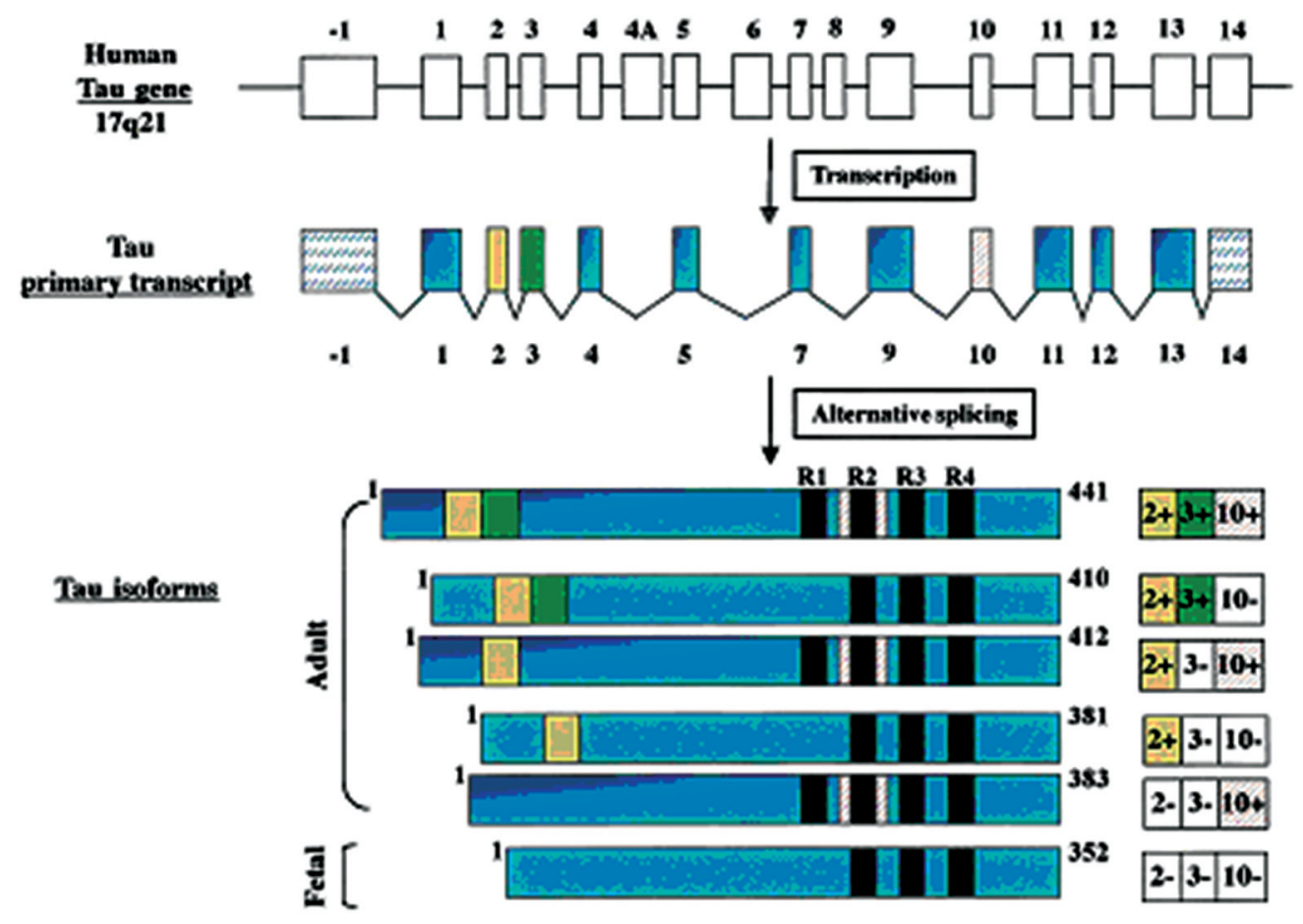

Figure 1. The tau gene, its primary transcript and the six tau isoforms that result from alternative splicing [1].

with vertical supranuclear ophthalmoplegia, and juvenile NPC disease [18]. The onset may be in infancy, early childhood, adolescence, or occasionally, in adulthood. Common neurological manifestations are clumsiness, ataxia, supranuclear gaze paresis, seizures and psychomotor retardation [19]. Brains from NPC patients exhibit neuronal distension in the cortex and swollen axons in the brainstem. In cases with a chronic progressive course, tau containing NFT are present in many parts of the brain, including the hippocampus, neocortex and several subcortical structures [20].

\section{THE TAU GENE AND PROTEIN}

The human tau gene extends over $100 \mathrm{~kb}$ on chromosome 17q21 and contains 16 exons. Restriction analysis and gene sequencing showed that it contains one $\mathrm{CpG}$ island associated with the promoter region, and another associated with exon 9 . The $\mathrm{CpG}$ island in the former region resembles those described in neuron-specific promoters [21-24]. The sequence of this region is TATA-less which indicates that it is likely to contain multiple initiation sites, as is typical of housekeeping genes [22]. Three SP1 (specificity protein 1)-binding sites that are important in directing transcription initiation in other TATA-less promoters are present in the proximity of the first transcription initiation site. The SP1-binding sites have been suggested to control neuronal specific expression of tau [12,21-24].

Three exons [4A, 6 and 8] are missing in the tau primary transcript expressed in the human brain and are specific to peripheral tau proteins. Exon $4 \mathrm{~A}$ occurs in bovine, human and rodent peripheral tissues with a high degree of homology [22]. Exon -1 (minus one) is part of the promoter and is transcribed but not translated. Exons 1, 4, 5, 7, 9, 11, 12 and 13 are constitutive exons. Exon 14 is part of the 3'UTR of tau mRNA. Exons 2, 3 and 10 are alternatively spliced and are adult brain-specific [21]. Exon 3 never appears independently of exon 2 . Thus, alternative splicing of these three exons allows for six combinations (2-3-10-; 2+3-10-; 2+3+10-; 2-3-10+; $2+3-10+; 2+3+10+)$ so that in the human brain, the tau primary transcript gives rise to six mRNAs (Fig. 1) $[21,22,25]$.

The human brain tau isoforms contain from 352 to 441 amino acids (molecular mass from 45 to 65 $\mathrm{kDa})$. They differ by the presence of either three (3R) 
or four (4R) repeat regions in the carboxy-terminal end and the absence or presence of one or two inserts ( 29 or 58 amino acids, respectively) in the aminoterminal end [12]. Since the isoforms are differentially expressed during development, each is likely to have a particular physiological role $[12,21]$. Only one, characterized by $3 \mathrm{R}$ and no amino-terminal inserts, is present during fetal stages, but all six isoforms are expressed during adulthood. Their specific functions may be related to the absence or presence of regions encoded by exons 2,3 and $10[21,26]$, and the isoforms may not be equally expressed in neurons. For instance, tau mRNAs containing exon 10 are not found in granular cells of the dentate gyrus. Thus, tau isoforms may be differentially distributed in neuronal subpopulations [12,21,26,27].

The presence or absence of 29 amino acid sequences encoded by exons 2 and 3 determine the length of the amino-terminal part of tau proteins [21]. These inserts are highly acidic and are followed by a basic proline-rich region (Fig. 2). The amino-terminal
Tau proteins bind to spectrin and actin filaments. Through these interactions, they may allow microtubules to interconnect with other cytoskeletal components, such as neurofilaments. There is evidence that tau also interacts with cytoplasmic organelles and the plasma membrane $[29,30,31]$.

Tau proteins bind to microtubules through the repeat domains (R1, R2, R3 and R4) encoded by exons 9-12 in their carboxy-terminal region. The $3 R$ or $4 \mathrm{R}$ copies of a highly conserved 18 -amino acid repeat are separated from each other by less conserved 13- or 14-amino acid inter-repeat domains. Tau proteins act as promoters of tubulin polymerization in vitro, and are involved in axonal transport [29]. The 18-amino acid repeats bind to microtubules through a variety of weak interactions. Adult tau isoforms with $4 \mathrm{R}$ are more efficient at promoting microtubule assembly than the fetal isoform with $3 \mathrm{R}[21,29]$. The most potent in inducing microtubule polymerization is the inter-region between R1 and R2 which is unique to 4R-tau. Recent evidence supports a role for

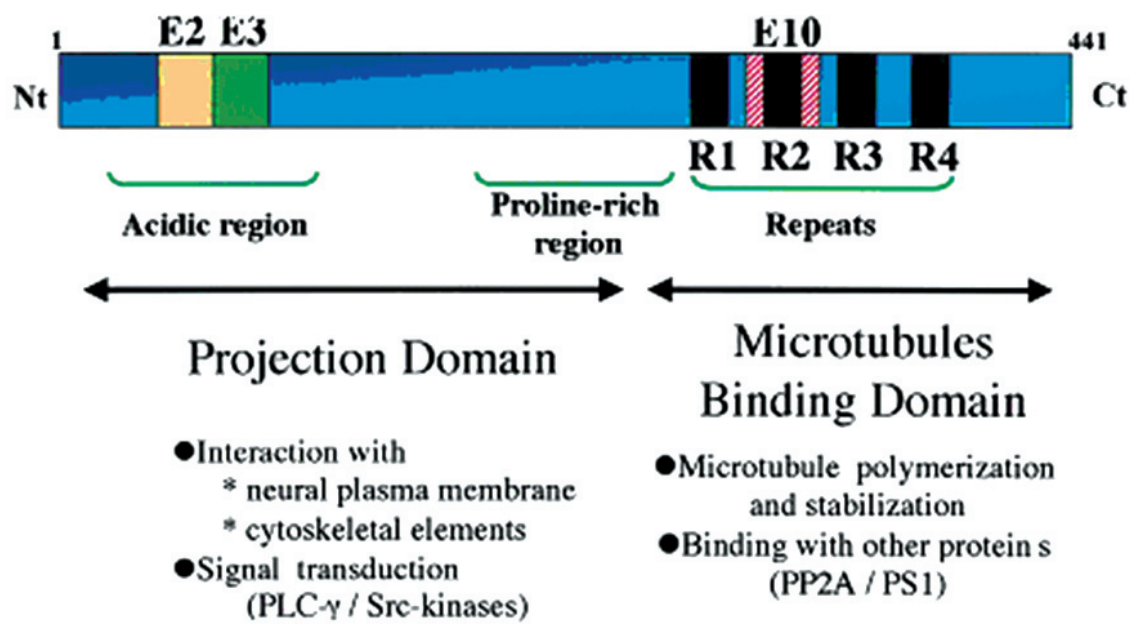

Figure 2. Projection and microtubule-binding domains of tau on the longest tau isoform. E: exon; R: repeat [1].

region is referred to as the projection domain, since it projects from the microtubule surface where it may interact with other cytoskeletal elements and with the plasma membrane [28]. In fact, projection domains of tau determine spacings between microtubules in an axon and may increase axonal diameter. In peripheral neurons, which often project a very long axon with a large diameter, an additional amino-terminal sequence encoded by exon $4 \mathrm{~A}$ is present, producing the specific isoform called "big tau" $[21,27,28]$. the microtubule-binding domain in the modulation of the phosphorylation state of tau proteins [21,30].

Posttranslational Modifications in Tau Proteins. One posttranslational modification observed in the tau protein is O-glycosylation [32]. The number of O-GlcNAcylated sites on tau protein is lower than the number of phosphorylation sites. Although the functional significance of O-GlcNAc modification is not fully understood, it is implicated in transcriptional regulation, protein degradation, cell activation, 
cell cycle regulation and the proper assembly of multimeric protein complexes $[32,33]$. O-GlcNAcylation may have a role in mediating interaction of tau with tubulin or in subcellular localization and degradation of tau [32,33-35].

Another posttranslational modification seen in tau is phosphorylation. There are 80 putative serine or threonine phosphorylation sites in the longest (441 amino acid) brain tau isoform [21]. Microtubule assembly depends partially upon the phosphorylation state, since phosphorylated tau is less effective than non phosphorylated tau in microtubule polymerization. In many neurodegenerative disorders, tau proteins aggregate into intracellular filamentous inclusions [36]. In AD, these inclusions are PHF, their constituent proteins are referred to as PHF-tau, and are found in a hyperphosphorylated state. There is a direct relationship between hyperphosphorylation, abnormal phosphorylation and tau aggregation, but it remains to be determined whether phosphorylation is a cause or a consequence of the aggregation process [36-38].

\section{CLASSIFICATION OF TAU AGGREGATION IN TAUOPATHIES}

In tauopathies other than $\mathrm{AD}$, abnormally and hyperphosphorylated tau protein aggregates are observed in the absence of amyloid deposits. Since tau aggregates differ in both degree of phosphorylation and content of tau isoforms, a molecular classification (depending on the molecular masses of the tau aggregates) into five classes has been proposed for the tauopathies (Fig. 3) [13,21].
Class 0: No Tau Aggregates. After AD, frontal lobe degeneration is the second most common pre senile dementia disorder in Europe [21]. It has a frontal pathology, like Pick's disease, but no specific neuropathological hallmark. Morphological changes comprise neuronal cell loss, spongiosis and gliosis, mainly in the superficial cortical layers of the frontal and temporal cortex. No tau aggregates are observed although loss of tau protein expression is demonstrated in this disorder, also named DLDH (dementia lacking distinctive histopathological) [21].

Class 1: A Major Tau Triplet of 60, 64, 69 kDa. This class is characterized by a pathological tau triplet of isoform of 60,64 , and $69 \mathrm{kDa}$ and a minor pathological tau of $72 / 74 \mathrm{kDa}$. This tau isoform triplet arises from aggregation among the six tau isoforms $[1,12,21]$. Thus, tau 60 is composed of the shortest tau isoform, and tau 64 and 69 are mixtures of tau isoforms with exon 10 or exon 2 alone, and exon $2+10+$ or exon $2+3+$, respectively. The longest tau isoform including exon $2+3+10+$ constitutes the $72 / 74 \mathrm{kDa}$ component. The prototypical neurodegenerative disorder that characterizes this class is AD, but this class also includes FTDP-17, ALS/PDC of Guam, postencephalitic parkinsonism, Down's syndrome and NPC $[1,21]$.

Class 2: A Major Tau Doublet of 64 and 69 kDa. This class is characterized by aggregation of 4R-tau isoform and is observed in PSP, CBD and FTDP-17. In both PSP and CBD, the pathological tau profile consists of the aggregation of 4R-tau isoforms, although a study of a large series of PSP

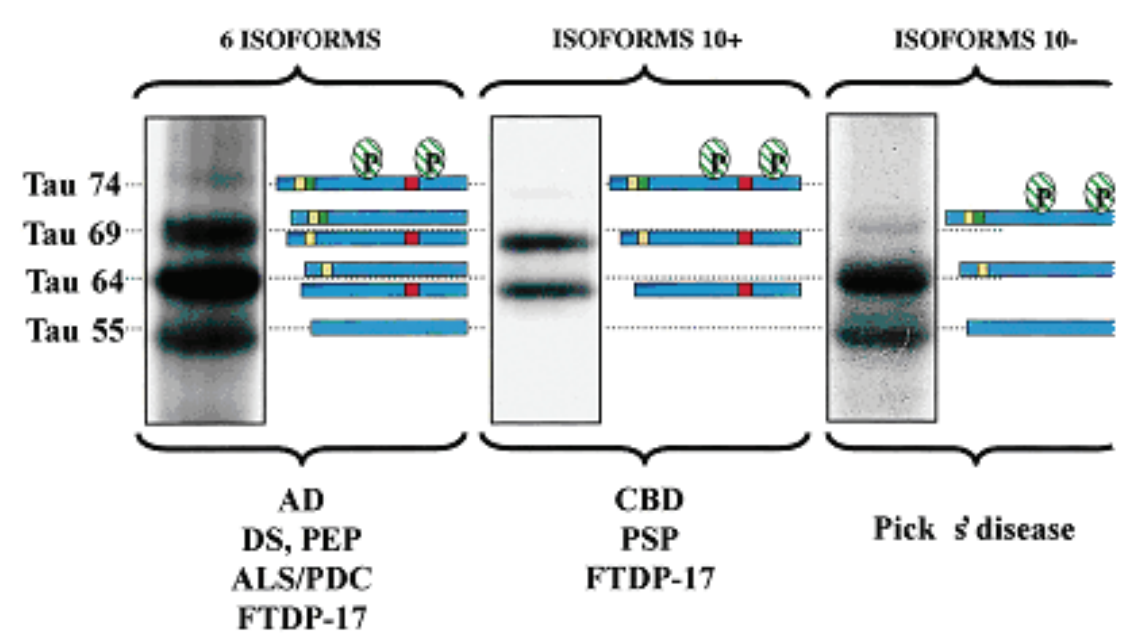

Figure 3. Electrophoretic profiles of pathological tau proteins with their molecular masses $(\mathrm{kDa})$, and classification of several tauopathies. Isoforms 10+: isoforms containing exon 10; isoforms 10-: isoforms without exon 10 [1]. 
patients revealed that the pathological tau profile is heterogeneous and includes variable amounts of $3 \mathrm{R}$ tau isoforms [21]. Thus, an increased ratio of $4 \mathrm{R} / 3 \mathrm{R}$ tau isoform is considered to define this class of tauopathies [11,13,21].

Class 3: A Major Tau Doublet of 60 and 64 kDa. In all cases of Pick's disease, the disorder characteristic of class 3, a major 60 and $64 \mathrm{kDa}$ tau doublet is observed. The tau profile of Pick's disease is the opposite of that of class 2. The pathological isoforms consist of the 3R-tau isoforms [21].

Class 4: A Major Tau of $60 \mathrm{kDa}$. This class consists only of DM type 1 (DM1). The tau profile of DM1 is characterized by a strong tau band of 60 $\mathrm{kDa}$ and to a lesser extent, of isoforms of 64 and 69 $\mathrm{kDa}$ [21]. This profile reflects a reduced number of isoforms expressed in the brain of DM1 patients, at both the protein and the mRNA levels. Using specific immunological probes against amino acid sequences in exon 2 and exon 3, the neurofibrillary lesions were shown to be devoid of tau isoforms with amino-terminal inserts [1,21]. An altered splicing of tau gene transcript is considered to be the cause, although the mechanism remains to be established. This demonstrates that the central nervous system is affected in DM1, making this a real tauopathy [21].

\section{TAU MUTATIONS/POLYMORPHISMS AND TAUOPATHIES}

As of 2004, 34 pathogenic tau mutations have been described in 101 FTDP-17 families [25]. They include 21 missense mutations, three silent mutations, two in-frame single codon deletions, and nine intronic mutations (Table 1). In addition, 17 coding polymorphisms have been reported [25]. The mutations are clustered in exons 9-13 which encode the microtubule-binding domain and flanking regions $[39,40]$. Most coding polymorphisms are within exons 4A, 6 and 8, that are not expressed in any of the major brain isoforms $[39,41,42]$. The most frequently observed mutations are a $\mathrm{C} \rightarrow \mathrm{T}$ substitution corresponding to $\mathrm{P} 301 \mathrm{~L}^{*}$ in exon 10 (identified in 25 families) and IVS-10+16 $(\mathrm{C} \rightarrow \mathrm{T})$ in intron 10 (identified in 22 families). In contrast, 21 mutations were reported in a single family [25]. Almost all mutations are heterozygous and segregate in a dominant manner within families; however, homozygous N296del* and S352L* mutations have been observed in siblings of consanguineous marriages $[25,43,44]$.

Mutations in tau proteins may affect alternative splicing of exon 10 and lead to changes in the proportion of 4R- and 3R-tau isoforms, or modify tau

Table 1. Tau gene mutations leading to FTDP-17 [25].

\begin{tabular}{|c|c|c|c|c|c|c|c|}
\hline Mutation & Location & Aggregate & Isoform & Mutation & Location & Aggregate & Isoform \\
\hline $\mathrm{R} 5 \mathrm{H}$ & exon 1 & glial & $4 \mathrm{R}$ & +3 & intron 10 & neuronal/glial & $4 \mathrm{R}$ \\
\hline R5L & exon 1 & neuronal & $4 R+3 R$ & +11 & intron 10 & neuronal/glial & $4 \mathrm{R}$ \\
\hline K257T & exon 9 & neronal & $3 \mathrm{R}>4 \mathrm{R}$ & +12 & intron 10 & neuronal/glial & $4 \mathrm{R}$ \\
\hline L266V & exon 9 & neuronal/glial & $3 R+4 R$ & +13 & intron 10 & neuronal/glial & $4 \mathrm{R}$ \\
\hline G272V & exon 9 & neuronal & $3 \mathrm{R}+4 \mathrm{R}$ & +14 & intron 10 & neuronal/glial & $4 \mathrm{R}$ \\
\hline $\mathrm{I} 260 \mathrm{~V}$ & exon 9 & $?$ & $?$ & +16 & intron 10 & neuronal/glial & $4 \mathrm{R}$ \\
\hline $\mathrm{N} 279 \mathrm{~K}$ & exon 10 & neuronal/glial & $4 \mathrm{R}$ & +19 & intron 10 & $?$ & $3 R>>4 R$ \\
\hline$\Delta \mathrm{K} 280$ & exon 10 & $?$ & $?$ & +29 & intron 10 & $?$ & $3 \mathrm{R}>>4 \mathrm{R}$ \\
\hline L284L & exon 10 & neuronal & $4 \mathrm{R} ?$ & +33 & intron 10 & $?$ & $?$ \\
\hline $\mathrm{N} 296 \mathrm{H}$ & exon 10 & neuronal/glial & $4 \mathrm{R}$ & L315R & exon 11 & $?$ & $?$ \\
\hline $\mathrm{N} 296 \mathrm{~N}$ & exon 10 & neuronal/glial & $4 \mathrm{R}$ & S320F & exon 11 & neuronal & $?$ \\
\hline$\Delta \mathrm{N} 296$ & exon 10 & $?$ & $?$ & V337M & exon 12 & neuronal & $3 R+4 R$ \\
\hline P301L & exon 10 & neuronal & $4 \mathrm{R}$ & E342V & exon 12 & neuronal & $4 \mathrm{R}$ \\
\hline P301S & exon 10 & neuronal & $4 \mathrm{R}$ & S352V & exon 12 & neuronal & $?$ \\
\hline S305N & exon 10 & neuronal & $4 \mathrm{R}$ & K369I & exon 12 & neuronal/glial & $3 R+4 R$ \\
\hline \multirow[t]{2}{*}{ S305S } & exon 10 & neuronal & $4 \mathrm{R}$ & G389R & exon 13 & neuronal & $4 \mathrm{R}>3 \mathrm{R}$ \\
\hline & & & & R406W & exon 13 & neuronal & $3 R+4 R$ \\
\hline
\end{tabular}

In the left column single letters correspond to amino acids. R: arginine; H: histidine; L: leucine; K: lysine; T: threonine; V: valine; G: glycine; I: isoleucine; N: asparagine; P: proline; S: serine; F: phenylalanine; M: methionine; E: glutamic acid; W: tryptophan; R stands for repeat(s) in the right column. 


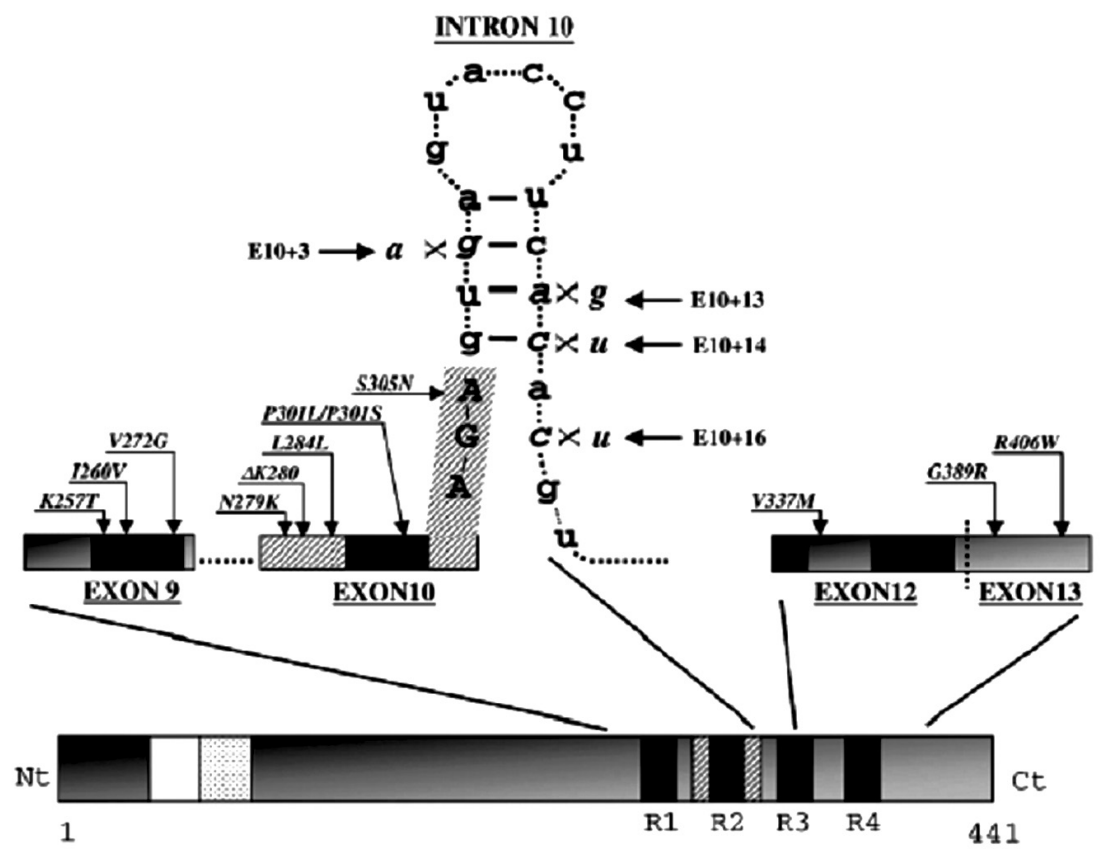

Figure 4. Stem loop structure of intron 10, mutations in intron 10 and exon 10, destabilizing this structure, and some other missense mutations in exons 9, 12 and 13 [1].

interactions with microtubules $[21,25]$. The former group includes intronic mutations $(+3,+13,+14$, $+16)$ and some missense mutations. Intronic mutations destabilize a stem loop structure in the 5' splice site of exon 10 that stabilizes this region of the premRNA [45-47]. Without this stem loop, access of U1snRNP to this site may be facilitated, increasing the formation of exon $10+$ tau mRNAs, and thus, the 4R-tau isoform [21]. In FTDP-17 families that carry these mutations, abnormally phosphorylated 4R-tau isoforms aggregate into filaments and display an electrophoretic profile similar to the major tau doublet of 64 and $69 \mathrm{kDa}$ seen in PSP and CBD [21]. Some missense mutations also modify the splicing of exon 10 (Fig. 4). The change in nucleotide that results in $\mathrm{N} 279 \mathrm{~K}^{*}$ and $\mathrm{S} 305 \mathrm{~N}^{*}$ mutations also creates an exon-splicing enhancer sequence. The silent mutation L284L* increases the formation of tau mRNAs containing exon 10 , presumably by destroying an exon-splicing silencing element. Families with one of these three missense mutations show the same tau electrophoretic profile as those having intronic mutations $[25,45]$. The latter group of tau mutations consists of missense mutations that affect microtubule assembly and polymerization. When located in the tau regions outside exon 10 they af- fect all tau isoforms so that none can properly bind to microtubules. These proteins aggregate into PHF and straight filaments in neuronal cells. Conversely, when these mutations are located in exon 10, 4R-tau isoforms are affected which do not bind to microtubules and aggregate into twisted ribbon filaments. This type of filamentous inclusion has been described in both neuronal and glial cells $[21,48,49]$.

Mutations of the tau gene and their involvement in FTDP-17 emphasize the fact that abnormal tau proteins may play a central role in the pathogenesis of neurodegenerative disorders, without involvement of the amyloid cascade [1,21]. The functional effects of the mutations suggest that a reduced ability of tau to interact with microtubules may be the first event upstream of hyperphosphorylation and aggregation. These mutations may also lead to an increase in free cytoplasmic tau (especially 4R-tau isoforms which facilitates their aggregation into filaments $[50,51]$.

Even before tau mutations were identified in FTDP-17 families, over representation of the most frequent allele (A0) of a dinucleotide repeat polymorphism, located in intron 9 of tau in PSP patients, had been reported [52]. This association has been confirmed in four independent populations [53-56]. Sequencing of the tau gene in FTDP-17 patients 
identified a series of polymorphisms within intronic sequences flanking tau exons $[25,54]$. Analysis of these polymorphisms revealed them to be in complete linkage disequilibrium with the intron 9 microsatellite, and led to identification of two major tau haplotypes ( $\mathrm{H} 1$ and $\mathrm{H} 2)$ extending over the complete coding tau region and the 5' promoter region of exon -1 (minus one) [7,25,57-59]. Recombination events between $\mathrm{H} 1$ and $\mathrm{H} 2$ have not been observed. Because the $\mathrm{A} 0$ allele is inherited as part of $\mathrm{H} 1$, increased frequencies of $\mathrm{H} 1$ in PSP populations have been identified $[52,53]$. Moreover, association studies in two CBD populations demonstrated similarly increased H1 frequencies, supporting a common genetic susceptibility for PSP and CBD [60,61]. Genetic investigation of the saitohin gene (STH) located within tau intron 9 , revealed a coding polymorphism changing a glutamine $(\mathrm{Q})$ to an arginine $(\mathrm{R})$ at codon 7 . Since the $\mathrm{Q}$ allele of STH is part of $\mathrm{H} 1$, it is no surprise that the positive association of PSP with tau is replicated by genotyping the Q7R polymorphism $[62,63]$.

In most populations, genotypic $(\mathrm{H} 1 \mathrm{H} 1)$ association is more significant than allelic (H1) association, suggesting the existence of a recessive mutation or a dosage-sensitive risk allele on H1 [25]. Both PSP and CBD are characterized by parkinsonism and neurodegeneration. Although they are pathologically distinct, similar tau deposits, predominantly composed of $4 \mathrm{R}$-tau isoforms, are found in the brains of these patients. It remains unclear how the presence of $\mathrm{H} 1$ would result in 4R-tau pathology. Although $\mathrm{H} 1$ and $\mathrm{H} 2$ differ within tau coding regions, missense mutations have only been identified in exons $4 \mathrm{~A}$ and 6 , which are not expressed in the major human brain isoforms. Since H1 is present in approximately $80 \%$ of the Caucasian population, extensive sequencing analysis of the $\mathrm{H} 1$ carriers is necessary to identify genetic variations that define disease-specific $\mathrm{H} 1$ subhaplotypes [25].

Tau association studies in other tauopathies (FTD, Pick's disease and AD) were negative or inconsistent in independent studies $[1,25]$. Although $\mathrm{H} 1 \mathrm{H} 1$ frequencies were found to be moderately increased in all FTD populations, they did not reach statistical significance [25]. The majority of tau association studies in AD populations were negative. Two studies, containing neuropathologically diagnosed Pick's disease patients, gave slightly increased association with the $\mathrm{H} 2 \mathrm{H} 2$ genotype $[64,65]$.

\section{CONCLUSIONS}

Aggregation of tau proteins in filamentous inclusions is a common feature of several neurodegenerative disorders. The laminar and regional distributions of NFT or other inclusions differ among conditions associated with dementia. The electrophoretic profiles of pathological tau proteins permit classification of these diseases on a basis of their biochemical signatures. These profiles may be explained by selective aggregation of specific sets of tau isoforms, differential vulnerability of neuronal subpopulations, and possibly variable sets of enzymes (kinases and/or phosphatases) that affect the phosphorylation state of the tau isoforms $[1,21,25]$.

Mapping of the spatiotemporal distribution of tau pathology in different brain areas is important in understanding how the disease spreads in the brain. While tau gene mutations simultaneously affect different brain areas, many sporadic tauopathies affect first a specific vulnerable area, e.g., the entorhinal cortex and hippocampal area in $\mathrm{AD}$, the brainstem in PSP and CBD. The extension of tauopathy in AD correlates with the evolution of cognitive deficits, from memory disorders to language impairment and then to apraxia and agnosia. It is interesting that the tau pathologies in PSP and CBD are quite different from those seen in $\mathrm{AD}$, since they emerge from subcortical nuclei toward the neocortex, especially toward frontal motor cortex [25]. Overall, the results discussed here, indicate that the key event in the tauopathies is disorganization of the cytoskeleton that leads to nerve cell degeneration, whether the primary cause is a tau gene mutation and/or polymorphism or some other unidentified factors.

\section{ACKNOWLEDGMENTS}

We would like to thank Boğaziçi University Research Funds, the State Planning Department of Turkey and Suna and Inan Kıraç Foundation for their generous financial support.

\section{REFERENCES}

1. Buée L, Bussiére T, Buée-Scherrer V, Delacourte A, Hof PR. Tau protein isoforms, phosphorylation and role in neurodegenerative disorders. Brain Res Rev, 2000; 33(1): 95-130. 
2. Iqbal K, Alonso AC, Chen S, Chohan MO, ElAkkad E, Gong CX, Khatoon S, Li B, Liu F, Rahman A, Tanimukai H, Grundke-Iqbal I. Tau pathology in Alzheimer disease and other tauopathies. Biochim Biophys Acta 2005; 1739(2-3): 198-210.

3. Hardy JA, Higgins GA. Alzheimer's disease: the amyloid cascade hypothesis. Science 1992; 256(5054): 184-185.

4. Buée-Scherrer V, Buée L, Leveugle D, Perl P, Vermesch P, Hof PR, Delacourte A. Pathological tau proteins in postencephalitic parkinsonism: comparison with Alzheimer's disease and other neurodegenerative disorders. Ann Neurol 1997; 42(3): 356-359.

5. Tolnay M, Probst A. The neuropathological spectrum of neurodegenerative tauopathies. Int Union Biochem Mol Biol Life 2003; 55(6): 299-305.

6. Albers DS, Augood SJ. New insights into progressive supranuclear palsy. Trends Neurosci 2001; 24(6): 347-352.

7. Molinuevo JL, Valldeoriola F, Alegret M, Oliva R, Tolosa E. Progressive supranuclear palsy: earlier age of onset in patients with the ô protein A0/A0 genotype. J Neurol 2000; 247(3): 206-208.

8. Stanford PM, Halliday GM, Brooks WS, Kwok JBJ, Storey CE, Creasey H, Morris JGL, Fulham MJ, Schofield PR. Progressive supranuclear palsy pathology caused by a novel silent mutation in exon 10 of the tau gene. Brain 2000; 123(5): 880-893.

9. Rojo A, Pernaute RS, Fontan A, Ruiz PG, Honnorat J, Lynch T, Chin S, Gonzalo I, Rabano A, Martinez A, Daniel S, Pramsteller P, Morris H, Wood N, Lees A, Tabernero C, Nyggard T, Jackson AC, Hanson A, de Yébenes JG. Clinical genetics of familial progressive supranuclear palsy. Brain 1999; 122(7): 1233-1245.

10. Higgins JJ, Rima L, Adler BA, Loveless JM. Mutational analysis of the tau gene in progressive supranuclear palsy. Neurology 1999; 2(7): 1421-1424.

11. Bergeron C, Davis A, Lang AE. Corticobasal ganglionic degeneration and progressive supranuclear palsy presenting with cognitive decline. Brain Pathol 1998; 8(2): 355-365.

12. Spillantini MG, Bird TD, Ghetti B. Frontotemporal dementia and parkinsonism linked to chromosome 17: a new group of taupathies. Brain Pathol 1998; 8(2): 387-402.

13. Buée L, Delacourte A. Comparative biochemistry of tau in progressive supranuclear palsy, corticobasal degeneration, FTDP-17 and Pick's disease.
Brain Pathol 1999; 9(4): 681-693.

14. Virginia $M$, Lee $Y$, Trojanowski JQ. Neurodegenerative tauopathies: human disease and transgenic mouse models. Neuron 1999; 24(3): 507-510.

15. Heutink P. Untangling tau-related dementia. Hum Mol Genet 2000; 9(6): 979-986.

16. Jaspert A, Fahsold R, Grehl H, Claus D. Myotonic dystrophy: correlation of clinical symptoms with the size of the CTG trinucleotide repeat. J Neurol 1995; 242(2): 99-104.

17. Hof PR, Bouras C, Perl DP, Sparks DL, Mehta $\mathrm{N}$, Morrison JH. Age-related distribution of neuropathologic changes in the cerebral cortex of patients with Down's syndrome. Arch Neurol 1995; 52(4): 379-391.

18. Loftus SK, Morris JA, Carstea ED, Gu JZ, Cummings C, Brown A, Ellison J, Ohno K, Rosenfeld MA, Tagle DA, Pentchev PG, Pavan WJ. Murine model of Niemann-Pick C disease: mutation in a cholesterol homeostasis gene. Science 1997; 277(5323): 232-235.

19. Love S, Bridges LR, Case CP. Neurofibrillary tangles in Niemann-Pick disease type C. Brain 1995; 118(1): 119-129.

20. Auer IA, Schmidt ML, Lee VMY, Curry B, Suzuki K, Shin RW, Pentchev PG, Carstea ED, Trojanowski JQ. Paired helical filament tau (PHF-tau) in Niemann-Pick tytpe C disease is similar to PHF-tau in Alzheimer's disease. Acta Neuropathol 1995; 90(6): 547-551.

21. Sergeant N, Delacourte A, Buée L. Tau protein as a differential biomarker of tauopathies. Biochim Biophys Acta 2005; 1739(2-3): 179-197.

22. Andreadis A, Wagner BK, Broderick JA, Kosik KS. A tau promoter region without neuronal specificity. J Neurochem 1996; 66(6): 2257-2263.

23. Heicklen-Klein A, Ginzburg I. Tau promoter confers neuronal specificity and binds Sp1 and AP-2. J Neurochem 2000; 75(4): 1408-1418.

24. Andreadis A, Brown WM, Kosik KS. Structure and novel exons of the human tau gene. Biochemistry 1992; 31(43): 10626-10633.

25. Rademakers R, Cruts M, van Broeckhoven C. The role of tau (MAPT) in frontotemporal dementia and related tauopathies. Hum Mutat 2004; 24(4): 277-295.

26. Goedert M, Spillantini MG, Jakes R, Rutherford D, Crowther RA. Multiple isoforms of human 
micro-tubule-associated protein tau: sequences and localization in neurofibrillary tangles of Alzheimer's disease. Neuron 1989; 3(4): 519-526.

27. Goedert M, Spillantini MG, Potier MC, U1rich J, Crowther RA. Cloning and sequencing of the cDNA encoding an isoform of microtubule-associated protein tau containing four tandem repeats: differential expression of tau protein mRNAs in human brain. EMBO J 1989; 8(2): 393-399.

28. Brandt R, Leger J, Lee G. Interaction of tau with the neural plasma membrane mediated by tau's amino-terminal projection domain. J Cell Biol 1995; 131(5): 1327-1340.

29. Hirokawa N, Shiomura Y, Okabe S. Tau proteins: the molecular structure and mode of binding on microtubules. J Cell Biol 1988; 107(4): 1449-1459.

30. Harada A, Oguchi K, Okabe S, Kuno J, Terada S, Ohshima T, Sato-Yoshitake R, Takei Y, Noda $\mathrm{T}$, Hirokawa N. Altered microtubule organization in small-calibre axons of mice lacking tau protein. $\mathrm{Na}-$ ture 1994; 369(6480): 488-491.

31. Dawson HN, Ferreira A, Eyster MV, Ghoshal N, Binder LI, Vitek MP. Inhibition of neuronal maturation in primary hippocampal neurons from tau deficient mice. J Cell Sci 2001; 114(6): 1179-1187.

32. Haltiwanger RS, Busby S, Grove K, Li S, Mason D, Medina L, Moloney D, Philipsberg G, Scartozzi R. O-glycosylation of nuclear and cytoplasmic proteins: regulation analogous to phosphorylation?. Biochem Biophys Res Commun 1997; 231(2): 237-242.

33. Kreppel LK, Blomberg MA, Hart GW. Dynamic glycosylation of nuclear and cytosolic proteins. J Biol Chem 1997; 272(14): 9308-9315.

34. Kamemura K, Hart GW. Dynamic interplay between O-glycosylation and O-phosphorylation of nucleocytoplasmic proteins: a new paradigm for metabolic control of signal transduction and transcription. Prog Nucleic Acid Res Mol Biol 2003; 73: 107-136.

35. Dong DL, Xu ZS, Hart GW, Cleveland DW. Cytoplasmic O-GlcNAc modification of the head domain and the KSP repeat motif of the neurofilament protein neurofilament-H. J Biol Chem 1996; 271(34): 20845-20852.

36. Lovestone S, Reynolds $\mathrm{CH}$. The phosphorylation of tau: a critical stage in neurodevelopment and neurodegenerative processes. Neuroscience 1997; 78(2): 309-324.

37. Seubert P, Mawal-Dewan M, Barbour R, Jakes R, Goedert M, Johnson GV, Litersky JM, Schenk D,
Lieberburg L, Trojanowski JQ. Detection of phosphorylated Ser262 in fetal tau, adult tau and paired helical filament tau. J Biol Chem 1995; 270(32): 18917-18922.

38. Goedert M, Hasegawa M, Jakes R, Lawler S, Cuenda A, Cohen P. Phosphorylation of microtubuleassociated protein tau by stress-activated protein kinases. FEBS Lett 1997; 409(1): 57-62.

39. Hayashi S, Toyoshima Y, Hasegawa M, Umeda Y, Wakabayashi K, Tokiguchi S, Iwatsubo T, Takahashi $\mathrm{H}$. Late-onset frontotemporal dementia with a novel exon 1 (Arg5His) tau gene mutation. Ann Neurol 2002; 51(4): 525-530.

40. Hasegawa M, Smith MJ, Iijima M, Tabira T, Goedert M. FTDP-17 mutations N279K and S305N in tau produce increased splicing of exon 10. FEBS Lett 1999; 443(2): 93-96.

41. Jiang Z, Cote J, Kwon JM, Goate AM, Wu JY. Aberrant splicing of tau pre-mRNA caused by intronic mutations associated with the inherited dementia frontotemporal dementia with parkinsonism linked to chromosome 17. Mol Cell Biol 2000; 20(11): 4036-4048.

42. Janssen JC, Warrington EK, Morris HR, Lantos P, Brown J, Revesz T, Wood N, Khan MN, Cipolotti L, Fox NC, Rossor MN. Clinical features of frontotemporal dementia due to the intronic tau $10(+16)$ mutation. Neurology 2002; 58(8): 1161-1168.

43. Hutton M. Missense and splice site mutations in tau associated with FTDP-17: multiple pathogenic mechanisms. Neurology 2001; 56(11 Suppl 4): S1-S25.

44. Poorkaj P, Grossman M, Steinbart E, Payami H, Sdaovnick A, Nochlin D, Tabira T, Trojanowski JQ, Borson S, Galasko D, Reich S, Quinn B, Schellenberg G, Bird TD. Frequency of tau gene mutations in familial and sporadic cases of non-Alzheimer dementia. Arch Neurol 2001; 58(3): 383-387.

45. Grover A, Houlden H, Baker M, Adamson J, Lewis J, Prihar G, Pickering-Brown S, Duff K, Hutton M. 5' Splice site mutations in tau associated with the inherited dementia FTDP-17 affect a stem-loop structure that regulates alternative splicing of exon $10 . \mathrm{J}$ Biol Chem 1999; 274(21): 15134-15143.

46. Spillantini MG, Murrell JR, Goedert M, Farlow MR, Klug A, Ghetti B. Mutation in the tau gene in familial multiple system tauopathy with presenile dementia. Proc Natl Acad Sci USA 1998; 95(13): 7737-7741. 
47. Varani L, Hasegawa M, Spillantini MG, Smith MJ, Murrell JR, Ghetti B, Klug A, Goedert M, Varani G. Structure of tau exon 10 splicing regulatory element RNA and destabilization by mutations of frontotemporal dementia and parkinsonism linked to chromosome 17. Proc Natl Acad Sci USA 1999; 96(14): 8229-8234.

48. Hasegawa M, Smith MJ, Goedert M. Tau proteins with FTDP-17 mutations have a reduced ability to promote microtubule assembly. FEBS Lett 1998; 437(3): 207-210.

49. Spillantini MG, van Swieten JC, Goedert M., Tau gene mutations in frontotemporal dementia and parkinsonism linked to chromosome 17 (FTDP-17). Neurogenetics 2000; 2(4): 193-205.

50. Yen S, Easson C, Nacharaju P, Hutton M, Yen SH. FTDP-17 tau mutations decrease the susceptibility of tau to calpain 1 digestion. FEBS Lett 1999; 461(1-2): 91-95.

51. Chiti F, Stefani M, Taddei N, Ramponi G, Dobson CM. Rationalization of the effects of mutations on peptide and protein aggregation rates. Nature 2003; 424(6950): 805-808.

52. Conrad C, Andreadis A, Trojanowski JQ, Dickson DW, Kang D, Chen X, Wiederholt W, Hansen L, Masliah E, Thal LJ, Katzman R, Xia Y, Saitoh T. Genetic evidence for the involvement of tau in progressive supranuclear palsy. Ann Neurol 1997; 41(2): 277-281.

53. Oliva R, Tolosa E, Ezquerra M, Molinuevo JL, Valldeoriola F, Burguera J, Calopa M, Villa M, Ballesta F. Significant changes in the tau A0 and A3 alleles in progressive supranuclear palsy and improved genotyping by silver detection. Arch Neurol 1998; 55(8): 1122-1124.

54. Bennett $\mathrm{P}$, Bonifati V, Bonuccelli U, Colosimo C, de Mari M, Fabbrini G, Marconi R, Meco, G, Nicholl DJ, Stocchi F, Vanacore N, Vieregge P, Williams AC. Direct genetic evidence for involvement of tau in progressive supranuclear palsy. Neurology 1998; 51(4): 982-985.

55. Morris HR, Janssen JC, Bandmann O, Daniel SR, Rossor, MN, Lees AJ, Wood NW. The tau gene A0 polymorphism in progressive supranuclear palsy and related neurodegenerative diseases. J Neurol Neurosurg Psychiatry 1999; 66(5): 665-667.

56. Higgins JJ, Golbe LI, de Biase A, Jankovic J, Factor SA, Adler RL. An extended 5'-tau susceptibility haplotype in progressive supranuclear palsy. Neu- rology 2000; 55(9): 1364-1367.

57. Hoenicka J, Perez M, Perez-Tur J, Barabash A, Godoy M, Vidal L, Astarloa R, Avila J, Nygaard $\mathrm{T}$, de Yebenes JG. The tau gene A0 allele and progressive supranuclear palsy. Neurology 1999; 1(2): 1219-1225.

58. Baker M, Litvan I, Houlden H, Adamson J, Dickson D, Perez-Tur J, Hardy J, Lynch T, Bigio E, Hutton M. Association of an extended haplotype in the tau gene with progressive supranuclear palsy. Hum Mol Genet 1999; 8(4): 711-715.

59. Morris HR, Khan MN, Janssen JC, Brown JM, Perez-Tur J, Baker M, Ozansoy M, Hardy J, Hutton M, Wood NW,Lees AJ, Revesz T, Lantos P, Rossor MN. The genetic and pathological classification of familial frontotemporal dementia. Arch Neurol 2001; 58(11): 1813-1816.

60. Houlden H, Baker M, Adamson J, Grover A, Waring S, Dickson D, Lynch T, Boeve B, Petersen RC, Pickering-Brown S, Owen F, Neary D, Craufurd D, Snowden J, Mann D, Hutton M. Frequency of tau mutations in three series of non-Alzheimer's degenerative dementia. Ann Neurol 1999; 46(2): 243-248.

61. Houlden H, Baker M, Morris HR, MacDonald N, Pickering-Brown S, Adamson J, Lees AJ, Rossor MN, Quinn NP, Kertesz A, Khan MN, Hardy J, Lantos PL, George-Hyslop P, Munoz DG, Mann D, Lang AE, Bergeron C, Bigio EH, Litvan I, Bhatia KP, Dickson D, Wood NW, Hutton M. Corticobasal degeneration and progressive supranuclear palsy share a common tau haplotype. Neurology 2001; 56(12): 1702-1706.

62. Conrad C, Vianna C, Freeman M, Davies P. Saitohin, a novel putative gene in the tau locus. Proc Natl Acad Sci USA 2002; 99(11): 7751-7756.

63. de Silva R, Hope A, Pittman A, Weale ME, Morris HR, Wood NW, Lees AJ. Strong association of the saitohin gene Q7 variant with progressive supranuclear palsy. Neurology 2003; 61(3): 407-409.

64. Russ C, Lovestone S, Baker M, PickeringBrown SM, Andersen PM, Furlong R, Mann D, Powell JF. The extended haplotype of the microtubule associated protein tau gene is not associated with Pick's disease. Neurosci Lett 2001; 299(1-2): 156-158.

65. Morris HR, Baker M, Yasojima K, Houlden H, Khan MN, Wood NW, Hardy J, Grossman M, Trojanowski J, Revesz T, Bigio EH, Bergeron C, Janssen JC, McGeer PL, Rossor MN, Lees AJ, Lantos PL, Hutton M. Analysis of tau haplotypes in Pick's disease. Neurology 2002; 59(3): 443-445. 